\title{
OVA12 promotes tumor growth by regulating p53 expression in human cancer cells
}

\author{
Renfeng Zhang ${ }^{1}$, Xicai Wu ${ }^{2}$, Xiangfeng Xia ${ }^{3}$, Asma Khanniche ${ }^{4}$, Feifei Song ${ }^{5}$, \\ Bingchang Zhang ${ }^{1}$, Ying Wang ${ }^{4}$ and Hailiang $\mathbf{G e}^{4}$ \\ ${ }^{1}$ Department of Laboratory Medicine, Shandong Provincial Hospital Affiliated to Shandong University, Jinan, China \\ ${ }^{2}$ Clinical Laboratory, People's Hospital of Rizhao, Rizhao, China \\ ${ }^{3}$ Department of Radiology, The Third People's Hospital of Rizhao, Rizhao, China \\ ${ }^{4}$ Shanghai Institute of Immunology, Shanghai Jiao Tong University School of Medicine, Shanghai, China \\ ${ }^{5}$ Department of Pathology, Tenth People's Hospital of Tongji University, Shanghai, China \\ Correspondence to: Bingchang Zhang, email: zhangbingchangb@163.com \\ Ying Wang, email: ywang@sibs.ac.cn \\ Hailiang Ge, email: Hailiangsjtu@163.com
}

Keywords: OVA 12, tumor growth, p53

Received: August 04, $2016 \quad$ Accepted: April 10, $2017 \quad$ Published: April 28, 2017

Copyright: Zhang et al. This is an open-access article distributed under the terms of the Creative Commons Attribution License 3.0 (CC BY 3.0), which permits unrestricted use, distribution, and reproduction in any medium, provided the original author and source are credited.

\section{ABSTRACT}

Ovarian cancer-associated antigen 12 (OVA12) was first identified in an ovarian carcinoma complementary DNA (CDNA) expression library and has been shown to play an important role in tumor growth. Here, we found that overexpression of OVA12 accelerated tumor growth in different tumor cells, whereas OVA12 depletion was associated with the opposite effect. Moreover, knocking down OVA12 led to a significant increase in the protein levels of p53, and the overexpression of OVA12 significantly decreased endogenous p53 levels. In addition, OVA12 stimulated p53 polyubiquitination and degradation by the proteasome and promoted tumor growth at least partially through the p53 pathway. Taken together, these results indicate that OVA12 is a negative regulator of p53 and that inhibition of OVA12 expression might serve as a therapeutic target to restore tumor suppression.

\section{INTRODUCTION}

Identification of appropriate tumor antigens is the first and most important step in the successful development of antigen-specific immunotherapy. Cancer-testis antigens (CTAgs) are expressed in a broad range of human tumors, but their normal expression is restricted to immunologically "privileged" tissues, including developing germ cells and trophoblastic tissues in the placenta [1]. To date, more than 100 CTAgs have been reported in the literature [2]. The widespread expression of CTAgs in tumors further supports their oncogenic activity, suggesting that they might serve as potential targets for tumor diagnosis, antigen-specific vaccination and antigendirected immunotherapy. Thus, the search for novel CTAgs has been a continuous task in the field of tumor immunology.
Using a serological analysis of recombinant cDNA expression libraries (SEREX), which is based on immunoscreening a cDNA library derived from an ovarian cancer patient, a novel gene named OVA12 (protein molecular weight $12 \mathrm{kDa}$ ) was identified $[3,4]$. The gene OVA12 is located on human chromosome 9q34.3 with an ORF of $345 \mathrm{bp}$ and encodes a 114-amino-acid protein. In our previous study (ref), we showed that this gene is highly expressed in tumors and that it accelerates in vitro and in vivo tumor development. Moreover, we found that expression of OVA12 protein could resist tumor cell apoptosis induced by 5 -fluorouracil through upregulation of Mcl-1 and survivin [4]. Our previous findings suggest that OVA12 is a novel CT antigen that functions as a positive regulator of tumor cell proliferation and consequently may serve as a valuable target for 
tumor diagnosis and therapy development. However, the molecular mechanisms of the involvement of OVA12 in the process of tumorigenesis remains unclear.

p53 is a tumor suppressor protein and a transcriptional regulator that plays an important role in cellular responses to various stress signals $[5,6]$. Biochemically, p53 acts as a transcription factor that can both activate and repress gene expression [7, 8]. As such, p53 protects cells from a variety of stress signals, such as DNA damage, oncogenic insults and nucleotide depletion, by activating the transcription of a panel of genes involved in cell cycle arrest and apoptosis in addition to repressing the genes involved in antiapoptosis and cell cycle progression [9-11]. The importance of p53 as a tumor suppressor is highlighted by the fact that approximately $50 \%$ of all human cancers carry inactivating mutations in the p53 gene [12]. In certain subtypes of cancer, such as triple-negative breast cancer, mutations can be found in up to $80 \%$ of samples [13].

p53 is primarily regulated at the level of protein stability by its interacting partner, MDM2 [14, 15]. MDM2 functions as an E3 ubiquitin ligase, which mediates p53 ubiquitination and proteasomal degradation in vivo [16]. In addition, MDM2 binds to p53 and hinders its transcriptional activity. The MDM2 gene has been shown to be amplified and/or overexpressed in several different cancer types [17]. Surprisingly, the frequency of amplification and/or overexpression of MDM2 is relatively low in various tumors with a wild-type p53 status $[18,19]$. These data suggest that other mechanisms might also regulate p53 levels.

In this study, we found that OVA12 promoted both cell proliferation in vitro and xenograft tumor growth in vivo. Moreover, loss of OVA12 resulted in a significant increase in p53 levels, and its overexpression significantly decreased endogenous p53 levels. Thus, OVA12 regulates cell proliferation and cell cycle progression in a p53-dependent manner. Collectively, these results reveal that OVA12 is a novel diagnostic and potential therapeutic target in cancer.

\section{RESULTS}

\section{OVA12 promotes cell proliferation in vitro}

The novel tumor antigen OVA12 was identified by immunoscreening a cDNA library derived from an ovarian cancer patient through a SEREX analysis. OVA12 has been shown previously to be overexpressed in diverse primary human tumor tissues and cell lines. To further determine the role of OVA12 in regulating cancer cell proliferation, we stably overexpressed OVA12 in Caski and ZR75-1 cells. As shown in Figure 1A, cell proliferation was significantly enhanced by ectopic expression of OVA12, which is consistent with the observations of SMMC7721 and HO8910 cells. Next, we used two shRNAs to stably knockdown OVA12 expression in Siha cells. The efficiency of OVA12 knockdown was shown in Table 1. Downregulation of OVA12 remarkably reduced the growth of Siha cells as measured by CCK-8 analysis (Figure 1B). Furthermore, a colony formation assay in soft agar showed that Caski cells stably overexpressing OVA12 produced more and larger colonies, whereas OVA12-depleted cells yielded fewer and smaller colonies than mock cells (Figure 1C). We further assessed the impact of OVA12 on cell apoptosis by FACS. As shown in Figure 1D, OVA12shRNA transfection greatly increased apoptosis in Siha cells.

\section{OVA12 promotes tumor growth in vivo}

Because OVA12 is highly expressed in tumors and promotes cell proliferation in vitro, we speculated that OVA12 might enhance tumorigenesis in vivo. To examine this hypothesis, we performed xenograft tumor assays using Siha cells and Caski cells stably transfected with OVA12shRNA or infected with lentivirus to induce overexpression of OVA12, respectively. The results showed that OVA12depleted cells formed smaller tumors than control cells (Figure 2A and 2B), whereas OVA12-overexpressing cells generated larger tumors (Figure 2D and 2E). Furthermore, we observed that tumors derived from OVA12-depleted cells displayed a lower Ki67 index compared to mock cells (Figure 2C), whereas tumors originating from cells with OVA12 overexpression showed higher malignancy and much stronger proliferation ability, as indicated by HE staining and immunostaining with Ki67 antibody (Figure 2F). Taken together, these results indicate that OVA12 is a novel tumor antigen endowed with tumor promoting properties and the ability to positively regulate tumor growth.

\section{OVA12 negatively regulates the tumor suppressor p53}

To investigate the molecular mechanism by which OVA12 promoted tumor growth, we tested several signaling transduction pathways that have been previously demonstrated to be critical in tumorigenesis. We found that OVA12 knockdown resulted in an increase in p53 and p21 protein levels (Figure 3A). We further examined several p53 downstream molecules using real-time PCR and found that the mRNA levels of p21, PUMA, P53R2, and Fas were upregulated in OVA12-knockdown Siha and MCF-7 cells (Figure 3B). By contrast, p53 and p21 protein levels decreased when OVA12 was overexpressed in Caski and ZR-75-1 cells (Figure 3D). Consistently, the mRNA levels of the target genes of p53, such as p21, PUMA, P53R2, and Fas were greatly decreased in OVA12-overexpressing Caski cells (Figure 3E). Additionally, we found no change in the mRNA levels of p53 in OVA12-knockdown and OVA12-overexpressing tumor cells, suggesting a posttranscriptional regulation of p53 by OVA12. We further examined the p53 transcriptional activity using a luciferase reporter system. As shown in Figure $3 \mathrm{C}$ and 3F, OVA12 knockdown significantly increased the luciferase activity, whereas overexpression of OVA12 led to attenuated 
A
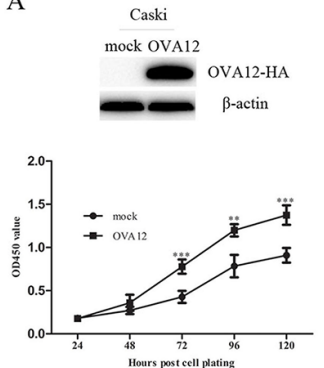

$\mathrm{C}$
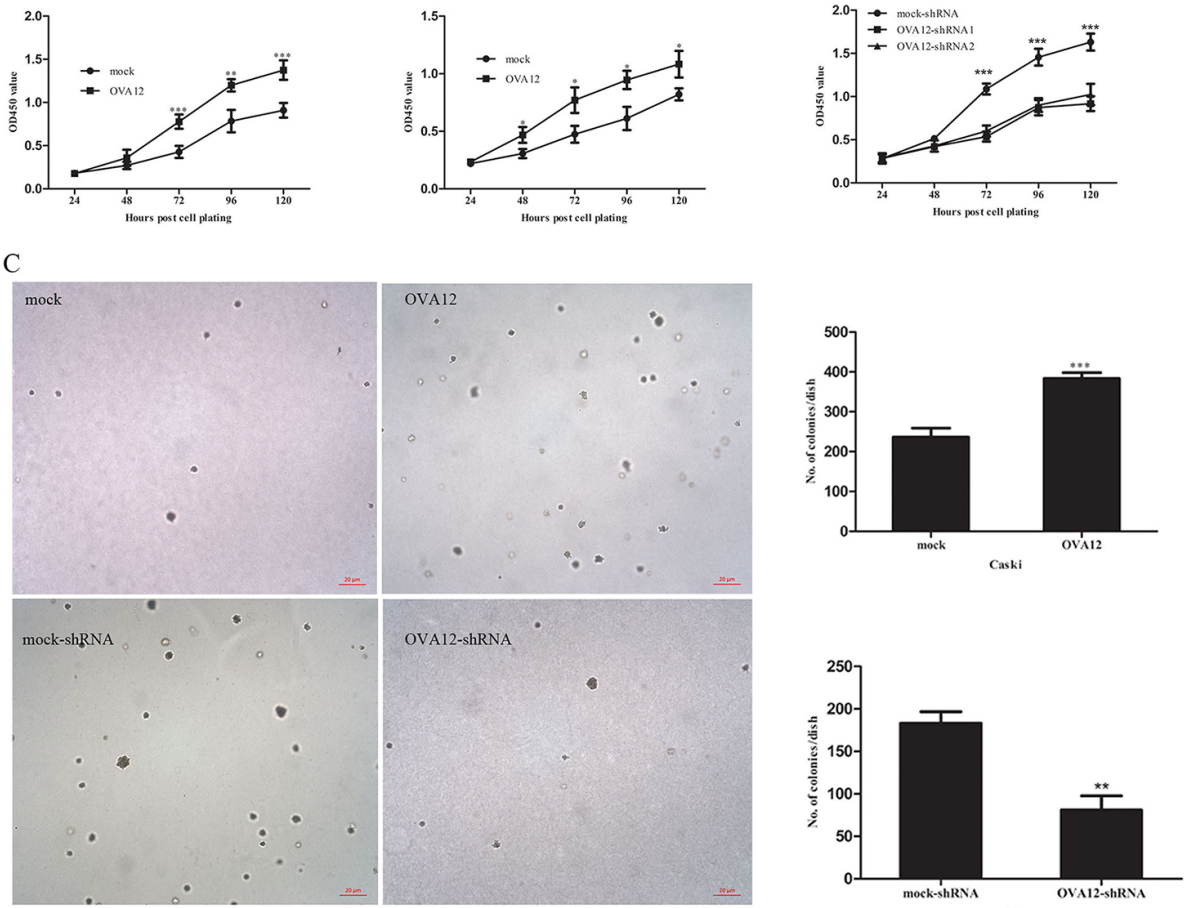

Siha

$\mathrm{D}$
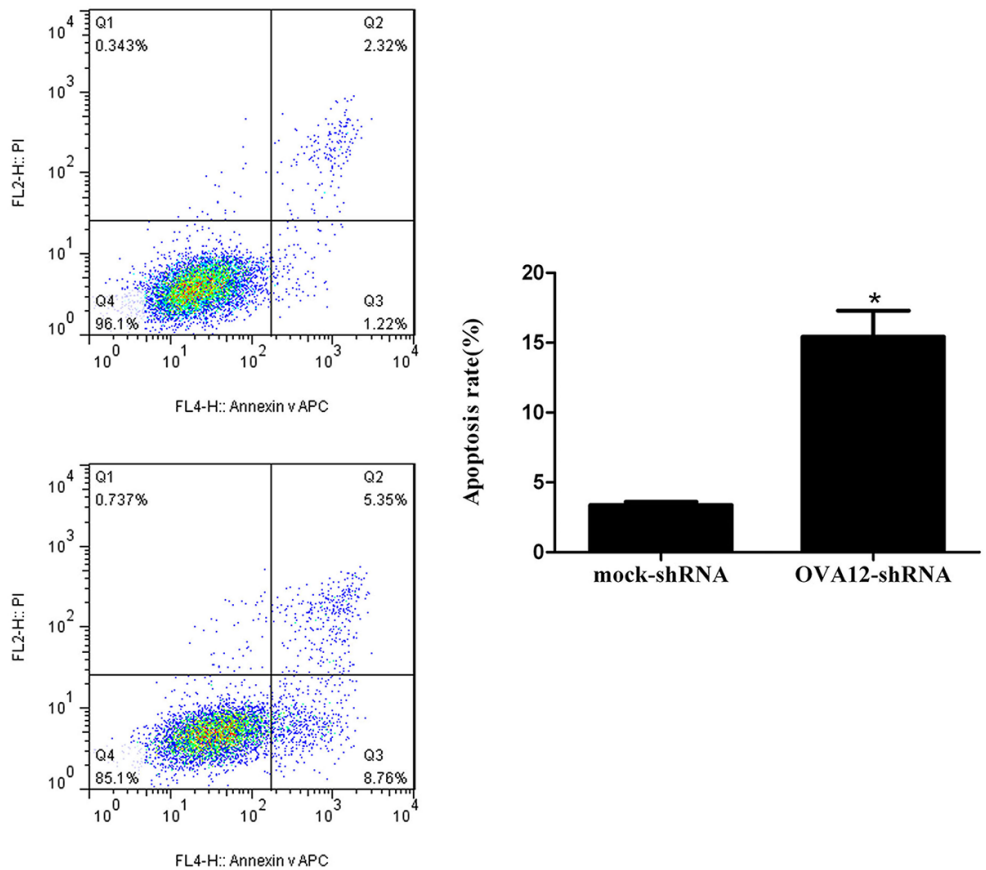

Figure 1: OVA12 promotes cell proliferation in vitro. (A) Overexpressoin of OVA12 enhances cell growth. Cell lines originating from the Caski and ZR-75-1 were established with stable overexpression of OVA12 and measured by CCK-8 assay. (B) Depletion of OVA12 suppresses cell growth. Cancer cells Siha were stably transfected with mock-shRNA and two OVA12-shRNAs and cell proliferation was examined by CCK-8 assay. (C) OVA12 promotes colony formation. Soft colony formation assay was performed using OVA12 genetransfected Caski cells and OVA12-shRNA-1 transfected Siha cells. (D) Down regulation of OVA12 induces apoptosis. The values shown are expressed as the mean $\pm \mathrm{SD}, \mathrm{n}=3,{ }^{*} \mathrm{p}<0.05$ (OVA12-shRNA group vs mock-shRNA group). 
Table 1: The efficiency of OVA12 knockdown in tumor cells

\begin{tabular}{lcccc}
\hline & \multicolumn{2}{c}{ CT value } & $\Delta \mathbf{C T}$ & $\Delta \Delta \mathbf{C T}$ \\
\hline Siha-mock-shRNA & $\boldsymbol{\beta}$-actin & OVA12 & OVA12-actin & \\
Siha-OVA12-shRNA1 & 14.22 & 21.88 & 7.66 & \\
Siha-OVA12-shRNA2 & 14.43 & 24.71 & 10.28 & 2.62 \\
\hline Relative & 14.33 & 24.16 & 9.83 & 2.17 \\
mRNA level & \multicolumn{2}{c}{ OVA12-shRNA1/mock-shRNA } & 0.163 \\
\hline
\end{tabular}

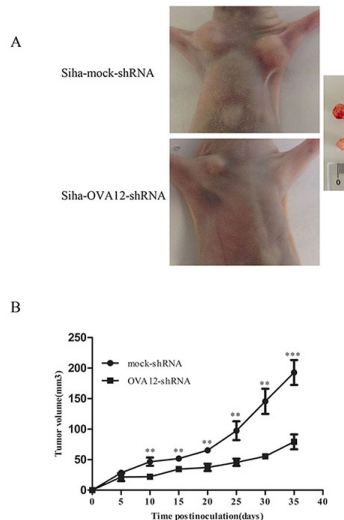

D

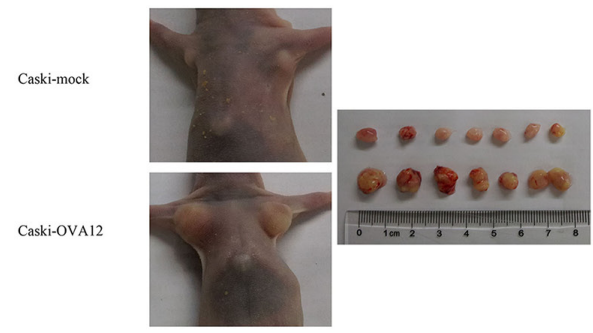

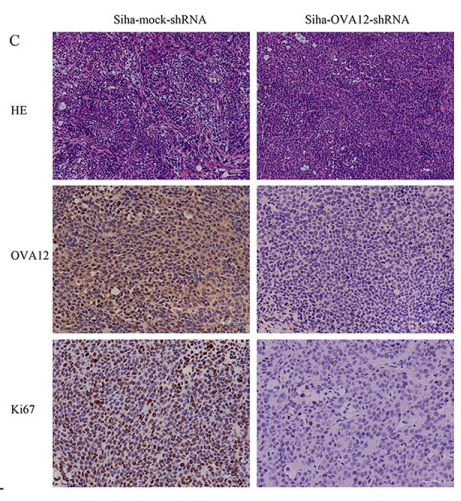

$\mathrm{E}$
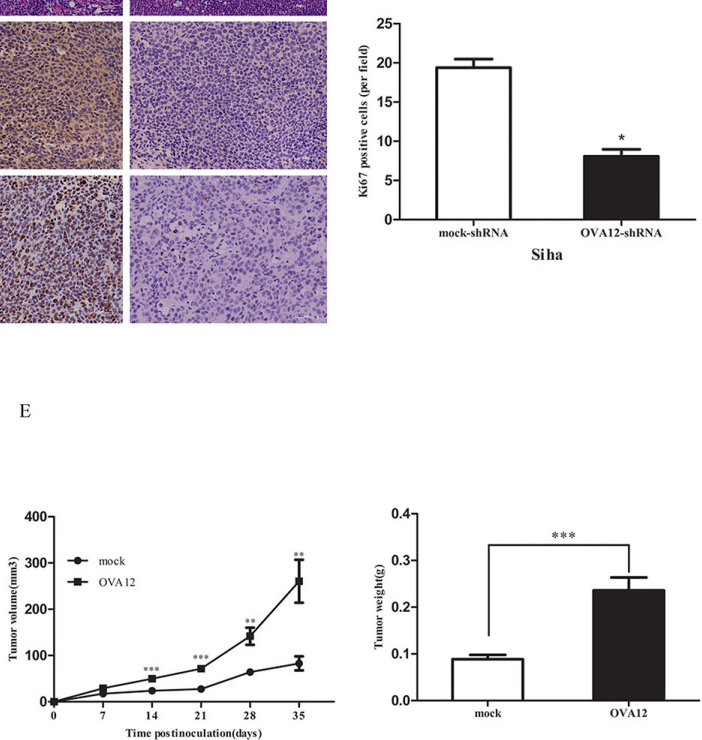

$\mathrm{F}$

OVA12

Ki67
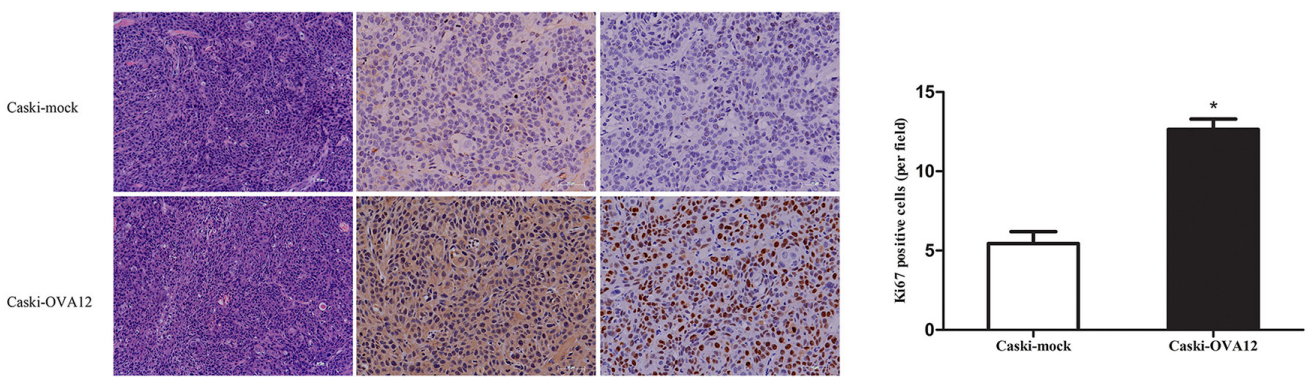

Figure 2: OVA12 promotes tumor growth in vivo. (A) Siha-OVA12-shRNA or Siha-mock-shRNA cells were subcutaneously injected into the nude mice. Images are of representative mice from each injection group and all the tumors of two xenografted groups. (B) Tumor volumes were monitored every 5 days for 35 days and tumor weights were measured. The values shown are expressed as the mean $\pm \mathrm{SD}, \mathrm{n}=7, * * \mathrm{p}<0.01,{ }^{* * *} \mathrm{P}<0.001$ (OVA12-shRNA group vs mock-shRNA group). (C) HE staining of the tumors and immunohistological staining of the tumors using an anti-OVA12 antibody and anti-Ki67 antibody. Xenograft tumors from Siha-OVA12-shRNA cells contain significantly less Ki67 positive proliferative cells. (D) Nude mice were injected subcutaneously with $5 \times 10^{6}$ cells per side for each of the indicated stable cell lines. The tumors were removed from nude mice and imaged. (E) Tumor growth curves and tumor weights after injection in nude mice. Tumor volumes were measured every week for 5 weeks $(n=7)$. Tumor weights were assessed at 35 days postinjection. The values shown are expressed as the mean $\pm \mathrm{SD}, \mathrm{n}=7, * * \mathrm{p}<0.01, * * * \mathrm{P}<0.001$ (OVA12 group vs mock group). (F) HE staining of the tumors and immunohistological staining of the tumors using an anti-OVA12 antibody and anti-Ki67 antibody. 
intensity of the p53 luciferase reporter. Consistently, we found significantly elevated expression of p53 and p21 in Siha OVA12-shRNA xenograft groups using IHC, and the reverse trend for p53 and p21 expression was observed in Caski OVA12-overexpressing xenograft groups (Figure 3G). We further examined the impact of OVA12 on mutant p53 in the MDA-MB-231 cell line carrying p53 mutation. As shown in Supplementary Figure 1A, overexpression of OVA12 did not affect the protein level of endogenous mutant p53.

\section{OVA12 enhances ubiquitination and degradation of p53}

Because we did not find any changes in p53 mRNA levels between OVA12-knockdown and OVA12-
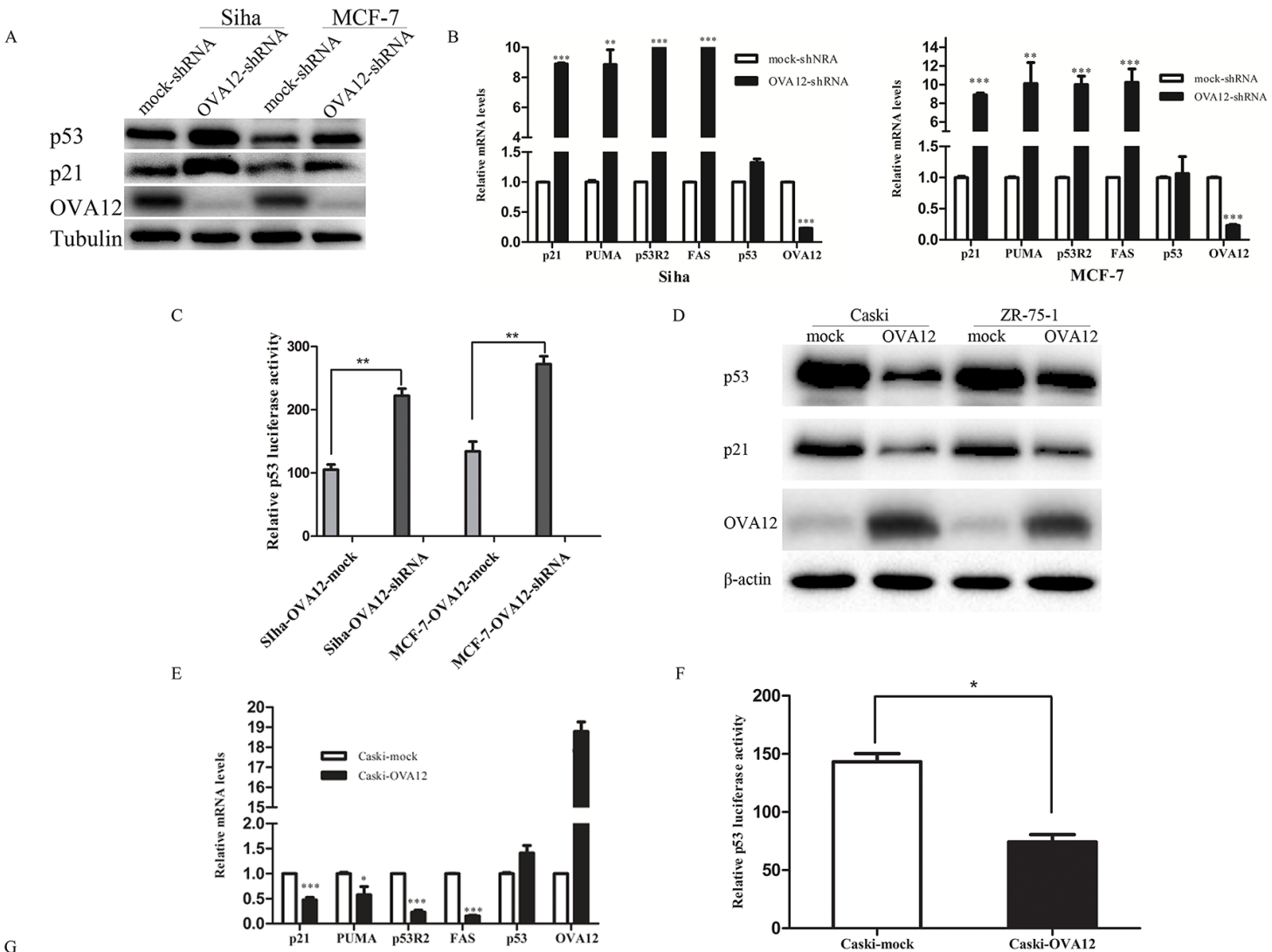

G
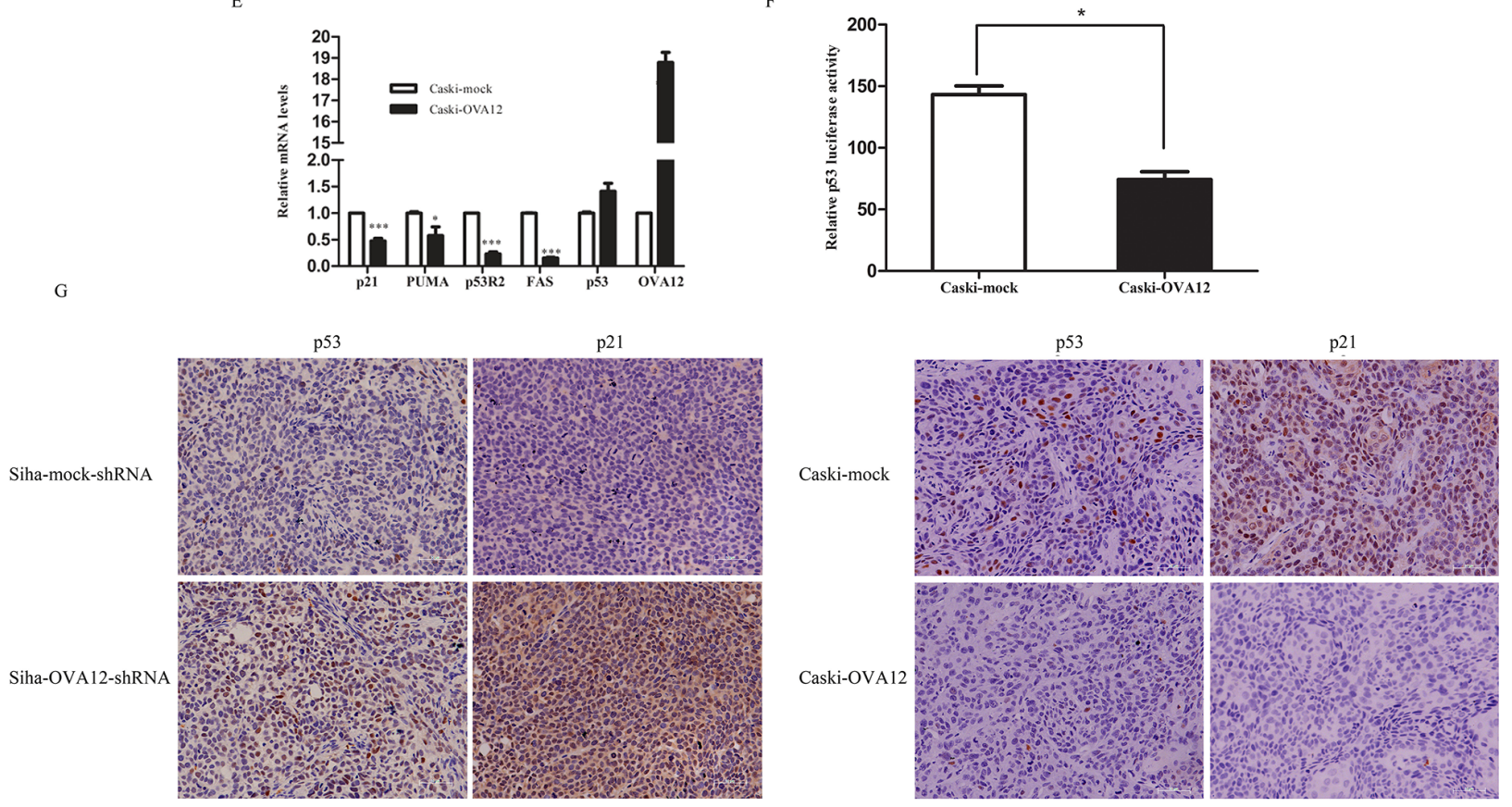

Figure 3: OVA12 negatively regulates p53 stability. (A) The shRNA-mediated depletion of OVA12 increases p53 and p21 protein levels in Siha and MCF-7 cells. (B) OVA12 knockdown increases the mRNA expression of p53 downstream target genes in Siha and MCF-7 cells. (C) Depletion of OVA12 promotes the luciferase activities of p53 reporter in Siha and MCF-7 cells. The values shown are expressed as the mean $\pm \mathrm{SD},{ }^{* *} \mathrm{p}<0.01$. (D and E) Overexpression of $O V A 12$ suppresses $\mathrm{p} 53$ and $\mathrm{p} 21$ protein levels and reduces the mRNA levels of $\mathrm{p} 53$ downstream genes in Caski and ZR-75-1 cells. (F) Overexpression of OVA12 inhibits the luciferase activities of p53 reporter in Caski cells. (G) IHC was performed to detect the p53 and p21 protein levels in xen-ograft tumors generated from Siha-mock-shRNA/OVA12-shRNA cells and Caski-mock/OVA12 cells. Representative pictures are shown at magnifications of $400 \times$. 
overexpressing tumor cells, we hypothesize that OVA12 promotes p53 protein polyubiquitination and proteasomedependent degradation. In agreement with our hypothesis, the results from co-immunoprecipitation experiments showed that ectopic expression of OVA12 dramatically increased the polyubiquitination of p53 in Caski and ZR75-1 cells (Figure 4A). By contrast, knockdown of OVA12 led to a decrease in the polyubiquitination of p53 in Siha and MCF-7 cells (Figure 4A).

Ubiquitin (Ub) chain extension can occur on a number of different lysine $(\mathrm{K})$ residues on the $\mathrm{Ub}$ molecule, but only chain polymerization at $\mathrm{K} 48$ is important for substrate degradation by the proteasome
$[20,21]$. To examine whether OVA12 promotes K48linked polyubiquitin chain formation of $\mathrm{p} 53$, we performed a ubiquitination assay with the ubiquitin mutant UbK48R, which cannot form K48-conjugated polyubiquitin chains. As shown in Figure 4B, OVA12 strongly induced the polyubiquitination of p53 in the presence of wild-type ubiquitin, but not K48R ubiquitin, suggesting that OVA12stimulated p53 polyubiquitination occurs predominantly on the K48 of Ub. These findings reveal that OVA12 promotes tumor growth by inducing ubiquitination and degradation of $\mathrm{p} 53$ by the proteasome.

We further examined the half-life of p53 in the presence of the protein translation inhibitor cycloheximide
A

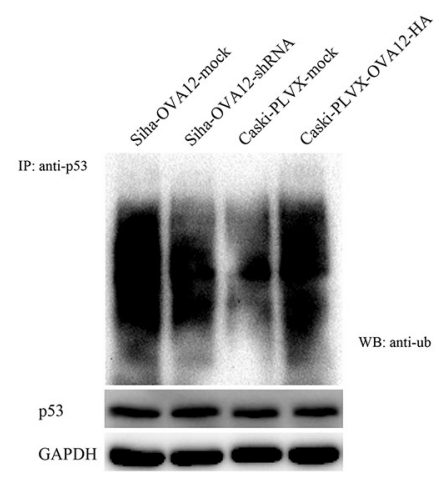

$\mathrm{C}$

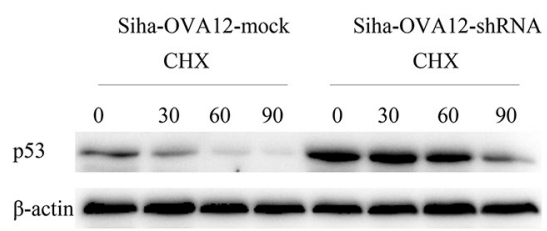

Caski-PLVX-mock

CHX

$\begin{array}{llllllll}0 & 30 & 60 & 90 & 0 & 30 & 60 & 90\end{array}$

p53

$\beta$-actin

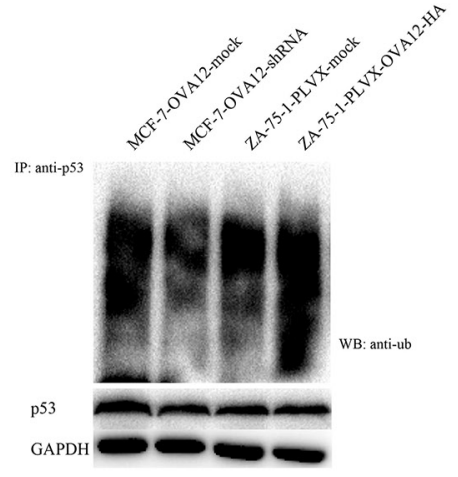

B

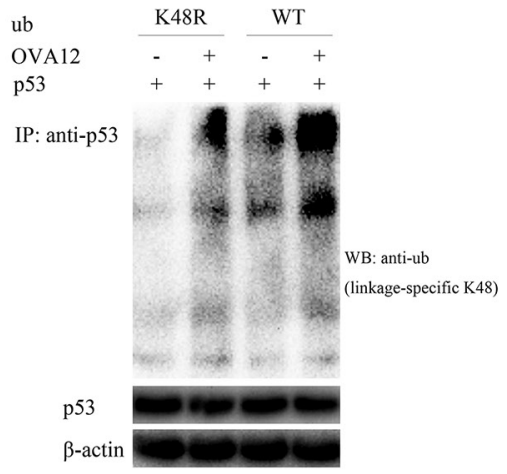

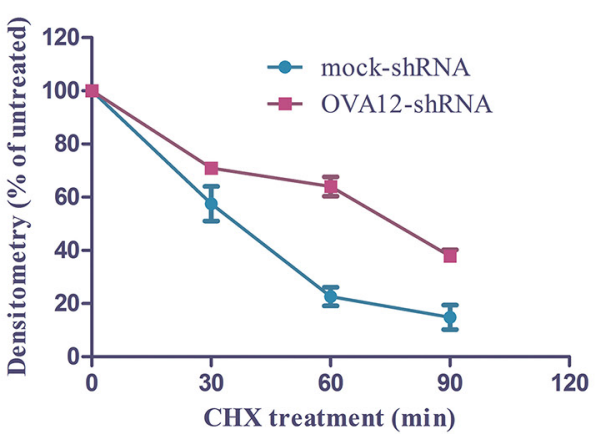

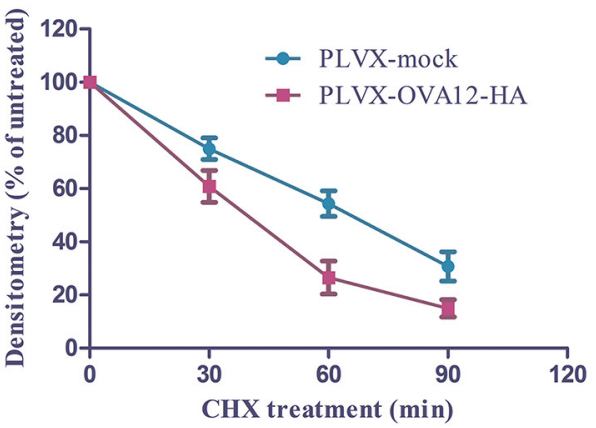

Figure 4: OVA12 promotes the polyubiquitination of p53. (A) Ubiquitination of 553 is enhanced by OVA12-overexpression while attenuated by OVA12 knockdown. (B) HO8910 cells were transfected with flag-OVA12, HA-UbWT, HA-UbK48R and pCMV/p53 in the indicated combinations. The cells were then treated with MG132 $(10 \mu \mathrm{M})$ for $5 \mathrm{~h}$. The cell lysates were subjected to immunoprecipitation followed by Western blot analysis. (C) Siha-mock-shRNA/OVA12-shRNA and Caski-mock/OVA12 cells were treated with CHX (25 $\mu$ g/ $\mathrm{ml}$ ) and harvested at indicated timepoints. The cell lysates were subjected to Western blot analysis. The data are represented as the mean \pm $\mathrm{SD}$ of the three independent experiments. 
(CHX) in Siha and Caski cells. When OVA12 was downregulated, the half-life of p53 was dramatically prolonged from $\sim 30$ to $\sim 70 \mathrm{~min}$. By contrast, ectopic expression of OVA12 noticeably shortened the half-life of p53 from $\sim 60$ to $\sim 30 \mathrm{~min}$ (Figure $4 \mathrm{C}$ ).

\section{The tumor promoting activity of OVA12 depends on p53}

To address the biological significance of p53 in the tumor-promoting function of OVA12, we investigated the influence of p53 knockdown on the tumorigenicity of OVA12 shRNA-transfected cells (Figure 5A). The depletion of OVA12 suppressed cancer cell proliferation, while p53 knockdown restored the cell growth rate resulting from the loss of OVA12 (Figure 5B). Cell cycle analysis using flow cytometry showed that knockdown of OVA12 caused G1 arrest in Siha cells, while silencing OVA12 and p53 together showed a marked reversal in G1 arrest (Figure 5C). Furthermore, tumor xenograft experiments also showed that the reduction of tumor volumes/weights by OVA12 downregulation was dependent on p53 function, as knocking down both OVA12 and p53 expression reversed tumor growth to the level of the control group (Figure 5D). These results strongly indicate that OVA12 promotes tumor cell growth at least partially through the p53 signaling pathway.
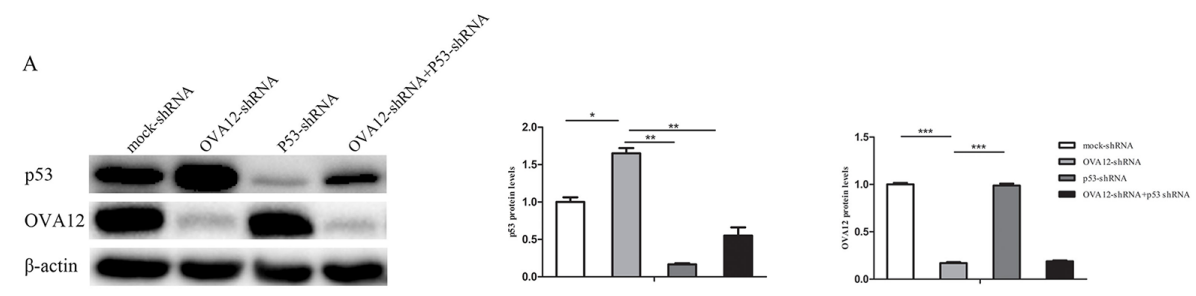

B

C
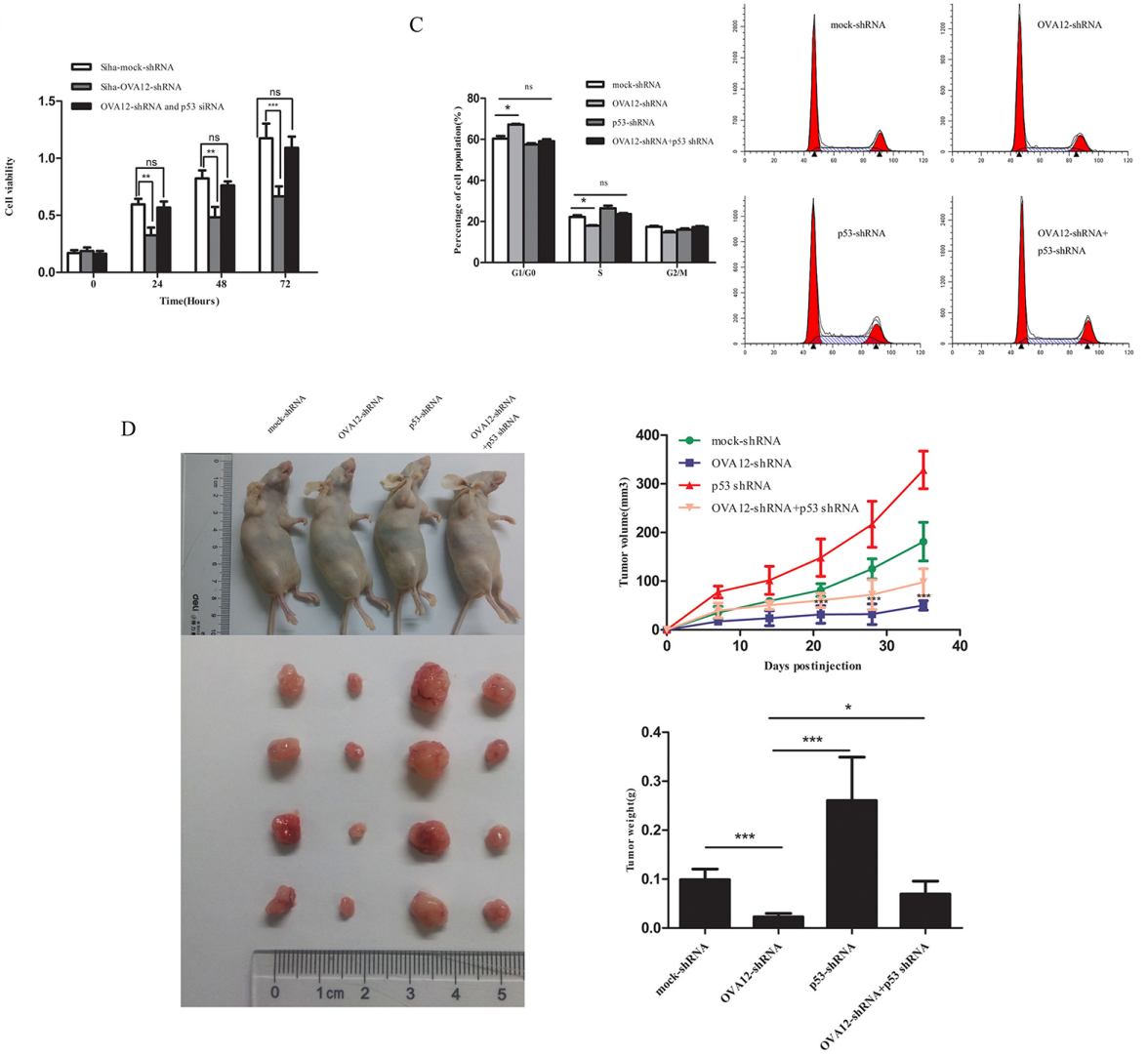

Figure 5: OVA12 exerts the tumor promoting activities through the p53 tumor suppressor. (A) Siha cells were transfected with OVA12-shRNA and p53-shRNA alone or in combination. The cell lysates were then subjected to immunoblot analysis. (B) p53 knockdown attenuates the inhibitory effect on the Siha cell proliferation by OVA12 knockdown. The cell proliferation ability was measured by CCK-8 assay. (C) Cell cycle analysis in Siha cells stably expressing the indicated shRNAs ( $\mathrm{n}=3$ ). (D) Siha cells stably expressing the indicated shRNAs were injected into nude mice. After 5-week injection, the mice were euthanized and analyzed for tumor growth. The values shown are expressed as the mean $\pm \mathrm{SD}, \mathrm{n}=4,{ }^{*} \mathrm{p}<0.05, * * * \mathrm{P}<0.001$ 


\section{OVA12 inhibits the expression of p14ARF and enhances the interaction between MDM2 and p53}

To further elucidate the mechanism by which OVA12 negatively regulates the p53 pathway, we performed an immunoprecipitation (IP) experiment and found that there was no interaction between OVA12 and p53 (data not shown). Simultaneously, we investigated the protein levels of MDM2, p-JNK and p14ARF and found that downregulation of OVA12 elevated the expression of p14ARF in Siha and MCF-7 cells, whereas overexpression of OVA12 inhibited the expression of p14ARF in Caski cells (Figure 6A). p14ARF can associate with MDM2 to inhibit the ubiquitination, nuclear export and subsequent degradation of $\mathrm{p} 53$. Because p14ARF physically sequesters MDM2 in nucleoli, thus relieving
A

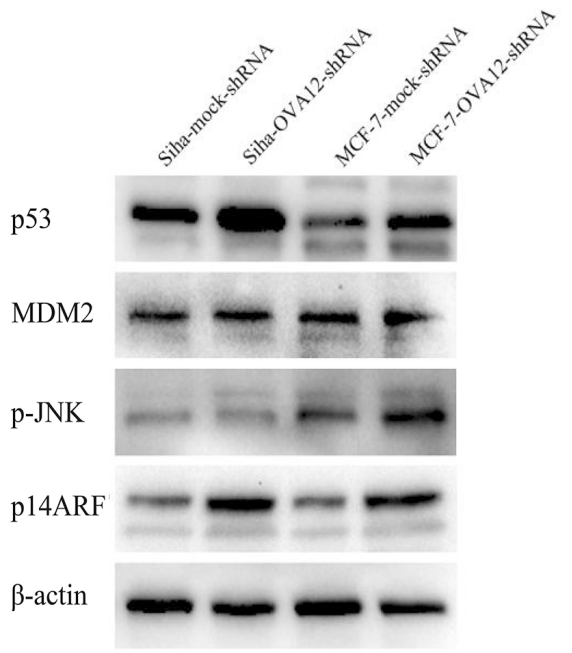

p53

MDM2

P-JNK

p14ARF

$\beta$-Aactin

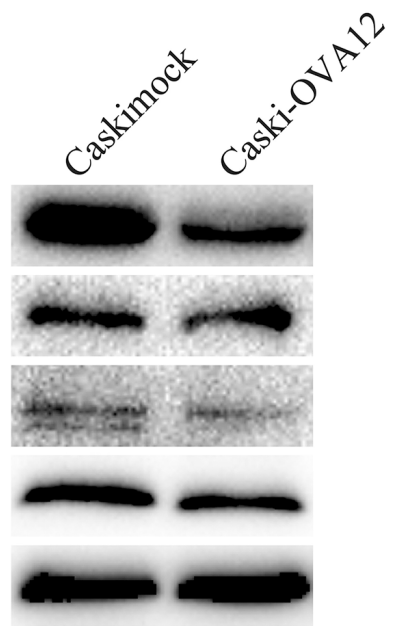

B

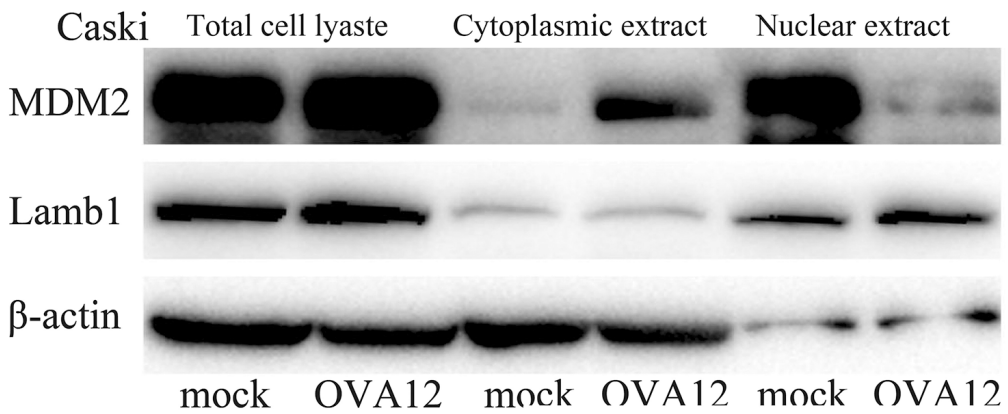

C

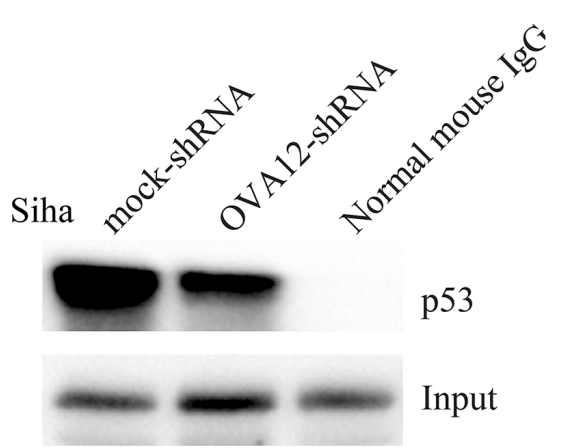

IP: anti-MDM2

IP: anti-MDM2

WB: anti-P53

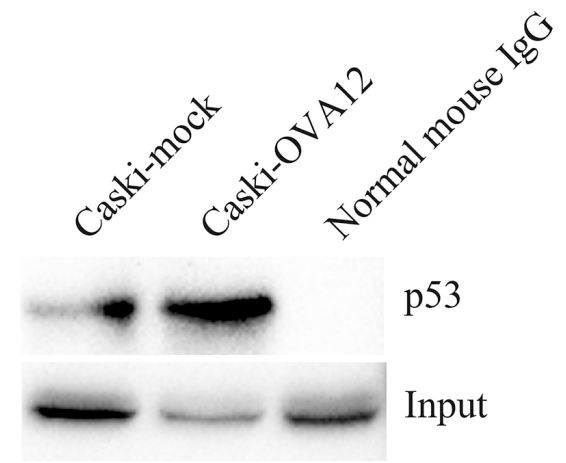

IB: anti-p53

Figure 6: OVA12 inhibits the expression of p14ARF and enhances the interaction between MDM2 and p53. (A) Downregulation of OVA12 elevated the expression of p14ARF in Siha and MCF-7 cells, whereas overexpression of OVA12 inhibited the expression of p14ARF in Caski cells. (B) OVA12 promoted the export of MDM2 from the nucleus to the cytoplasm. (C) Coimmunoprecipitation analysis of the protein interaction between MDM2 and p53 showed that overexpression of OVA12 enhanced the interaction between MDM2 and p53. 
Table 2: Primer sequences used in real-time PCR assay

\begin{tabular}{lll}
\hline Gene & Forward & Reward \\
\hline p21 & TTAGCAGCGGGACAAGGAGT & CGTTAGTGCCAGGAAAGACA \\
PUMA & AAAGGCTGTTGTGCTGGTG & TTTGGCTCATTTGCTCTTCA \\
p53R2 & CGGTTTGTCATCTTTCCAATC & GCTTGTTCCAGTGAGGGAGA \\
FAS & GGACCCTCCTACCTCTGGTT & TCCTCAATTCCAATCCCTTG \\
p53 & GCCATCTACAAGCAGTCACAG & ATTTCCTTCCACTCGGATAAGA \\
OVA12 & TGGGTGTTGCTGTGTATTCC & TCTTAGGTTCCAGGCAGTTCA \\
\hline
\end{tabular}

nucleoplasmic p53 from MDM2-mediated degradation, we examined the distribution of MDM2 in the cytoplasm and nucleus in Caski cells. We observed that OVA12 promoted the export of MDM2 from the nucleus to the cytoplasm (Figure 6B). Furthermore, co-immunoprecipitation analysis of the protein interaction between MDM2 and p53 showed that overexpression of OVA12 enhanced the interaction between MDM2 and p53 (Figure 6C).

\section{DISCUSSION}

Characterization of new tumor antigens is an active field in tumor immunology. OVA12 was first identified from an ovarian cancer cDNA expression library through a SEREX approach. A previous study revealed that OVA12 is highly expressed in a variety of tumors and exhibits tumor-growth promoting properties in SMMC7721 and HO8910 cells [4]. Here, we found that OVA12 also regulated tumorigenesis in other tumor cells, such as Caski, ZR-75-1 and Siha cells. Overexpression of OVA12 promoted Caski cell proliferation both in vitro and in vivo, whereas stable knockdown of OVA12 in Siha cells inhibited cell proliferation and xenograft tumor growth. Considering the critical role played by the tumor suppressor p53 in cell growth arrest and apoptosis, we investigated whether OVA12 affected p53 regulation. Interestingly, we found that knocking down OVA12 resulted in a significant increase in the p53 level and a reduction of endogenous p53 ubiquitination. Consistently, overexpression of OVA12 led to enhanced p53 ubiquitination and a decreased endogenous p53 steady-state level. In addition, OVA12 overexpression led to a significant decrease in the p53 protein half-life, whereas $O V A 12$ gene silencing resulted in a prolonged stability of p53.

Next, we investigated whether blocking p53 expression can rescue cell proliferation and growth arrest caused by the loss of OVA12 in Siha cells. The results showed that simultaneous inhibition of OVA12 and p53 expression by RNAi successfully rescued the OVA12depletion phenotype, indicating that OVA12 promoted tumorigenesis through downregulation of p53. However, we did not observe a full rescue. Therefore, we speculate that p53 is only partially responsible for the tumor promoting function of OVA12 antigen. Therefore, we do not exclude other targets that OVA12 might affect in the process of tumor development.

How does OVA12 promote p53 ubiquitination? The hypothesis and molecular mechanisms underlying this process have not been previously investigated. In this study, we found that OVA12 does not physically interact with p53 (data not shown). Interestingly, we observed that OVA12 overexpression downregulated the p14 ${ }^{\mathrm{ARF}}$ levels, whereas knockdown of OVA12 increased the $\mathrm{p} 14^{\mathrm{ARF}}$ levels. The $\mathrm{p} 14^{\mathrm{ARF}}$ tumor suppressor is a product of the INK4a/ARF locus and is a critical regulator of p53 stability [22]. p14 ${ }^{\mathrm{ARF}}$ can interact with MDM2 to inhibit the ubiquitination, nuclear export and subsequent degradation of p53 [23-32]. In our study, we found that overexpression of OVA12 promoted the export of MDM2 from the nucleus to the cytoplasm in Caski cells and promoted the interaction between MDM2 and p53. Whether OVA12 promoted p53 ubiquitination through the p14 ${ }^{\mathrm{ARF}}$-p53 pathway is an interesting question that should be explored in the future.

\section{MATERIALS AND METHODS}

\section{Cell cultures}

Cell lines (Siha, MCF-7,Caski, ZR-75-1) were obtained from the Shanghai Institutes for Biological Sciences, Chinese Academy of Sciences. Siha and Caski cells were maintained in Dulbecco's modified Eagle's minimal essential medium with $10 \%$ fetal bovine serum, and MCF-7 and ZR-75-1 cells were cultured in Roswell Park Memorial Institute 1640 medium with 10\% fetal bovine serum. All cell lines were cultured at $37^{\circ} \mathrm{C}$ in a humidified incubator with $5 \% \mathrm{CO}_{2}$.

\section{Plasmid construction and establishment of OVA12-overexpressing cells}

The cDNA of OVA12 was amplified by RT-PCR using the gene-specific primers 5'-atcgGTCGACGCC ACCATGTGGGCCCAGCCCTGTGCCA-3' (Forward) 
and 5'- atcgGCGGCCGCTCATTAGGTTCCAGGCAG TTCAAAAACA-3' (Reverse). The PCR products were cloned into the lentiviral expression vector pLVXpuro. Lentiviruses were produced by co-transfection of $293 \mathrm{~T}$ cells with pLVX-mock and pLVX-OVA12 vectors and packaging vectors pSAX2 and pMD2G using Lipofectamine 2000 (Invitrogen). The viral supernatant was collected $48 \mathrm{~h}$ post-transfection and used to infect Caski and ZR-75-1 cells. After $48 \mathrm{~h}$ of infection, the infected cells were then selected with $4 \mu \mathrm{g} / \mathrm{ml}$ puromycin for a continuous 4-day period to generate stable transfected cell lines.

\section{Establishment of stable OVA12-knockdown cells}

Based on the OVA12 cDNA sequence, two pairs of short hairpin RNA (shRNA) candidates were designed and introduced into a pSIREN expression vector, and they were named OVA12shRNA1(GGAATAAAGGTACATATAGAG) and OVA12shRNA2(GGTGTTGCTGCGTATTCCTGC). To package the virus, $293 \mathrm{~T}$ cells were seeded in a 6-well plate $24 \mathrm{~h}$ prior to transfection. Either OVA12 shRNA or mockshRNA was co-transfected with the packaging plasmid VSV-G and gag/pol using Lipofectamine 2000. At $48 \mathrm{~h}$ post-transfection, the viral supernatant was collected to infect target cell lines. After $48 \mathrm{~h}$ of infection, the infected cells were selected by exposure to $4 \mu \mathrm{g} / \mathrm{ml}$ puromycin for 4 days. Puromycin-resistant cells were isolated, cultured in medium containing $2 \mu \mathrm{g} / \mathrm{ml}$ puromycin and expanded for subsequent study.

\section{Cell proliferation assay}

Cells $\left(5 \times 10^{3}\right.$ per well $)$ were plated in 96 -well plates and cultured in medium with $0.5 \%$ FBS for $24 \mathrm{~h}$. Then, at specific time points $(24,48,72$, and $96 \mathrm{~h}), 10 \mu \mathrm{l}$ of CCK-8 solution was added, and the cells were incubated at $37^{\circ} \mathrm{C}$ for $2 \mathrm{~h}(\mathrm{n}=6)$. Absorbance was measured at $450 \mathrm{~nm}$ using a spectrophotometer.

\section{Soft colony formation assay}

Cells were suspended at a density of $2 \times 10^{3}$ per well in medium containing $10 \% \mathrm{FBS}$ and $0.3 \%$ low melting temperature agarose and placed on the top of solidified $0.7 \%$ agarose containing complete medium in a 6 well plate. After 2 weeks of culture, colonies were stained with $0.5 \%$ crystal violet and counted.

\section{Xenograft transplantation and in vivo tumor studies}

Cells were resuspended in $100 \mu \mathrm{l}$ of PBS and injected subcutaneously in both posterior flanks of 4 -week-old female athymic nude mice $\left(5 \times 10^{6}\right.$ cells per side). The size of the tumors was measured weekly using a caliper, and the tumor volume was calculated using the following formula: $V=$ length $\times$ width $^{2} \times 0.5$. The animals were sacrificed after 5 weeks, and the tumor bulk was harvested. All mice (Shanghai SLAC Laboratory Animal Co. Ltd., Shanghai, China) were housed in laminar flow cabinets under specific pathogen-free conditions. The study protocol was approved by the China Institutional Ethics Review Committee for Animal Experimentation.

The tumors were fixed with $4 \%$ buffered formalin, paraffin-embedded, and cut into $4-\mu \mathrm{m}$ sections for immunohistochemical staining of OVA12, Ki67, p53 and p21 expression. Antibodies used to detect Ki67, p53 and p21 were from Santa Cruz Biotechnology; the OVA12 antibody for IHC detection was generated by our lab. Secondary antibodies against mouse or rabbit immunoglobulin G were supplied in an IHC kit from Gene Tech Company Limited (Shanghai, China).

\section{Western blot analysis}

Cells were lysed on ice for $10 \mathrm{~min}$ with M-PERTM Mammalian Protein Extraction Reagent (Pierce), and the lysates were centrifuged at $14,500 \mathrm{rpm}$ at $4{ }^{\circ} \mathrm{C}$ for $15 \mathrm{~min}$. The protein concentration of the supernatant was determined using a BCA Protein Assay Reagent Kit (Pierce). Twenty micrograms of protein was separated with 12\% SDS-polyacrylamide gel electrophoresis and transferred onto a polyvinylidene difluoride membrane. Membranes were blocked for $1 \mathrm{~h}$ at room temperature and then incubated with primary antibodies overnight at $4{ }^{\circ} \mathrm{C}$. The membranes were then washed three times with TBST for $5 \mathrm{~min}$, blotted with hoseradish peroxidase (HRP)-conjugated secondary antibody (KPL company) for $1 \mathrm{~h}$ at room temperature and detected with enhanced chemiluminescence reagent (MILLIPORE). The following primary antibodies were used: anti-p53 (\#sc-926), antiMDM2 (\#sc-965), anti-p21 (\#sc-817), anti-ubiquitin (\#sc-8017) and anti-p-JNK (\#sc-6254, Santa Cruz); antiB-actin (Catalog \#3598-100, BioVision); Anti-Ubiquitin (linkage-specific K48) antibody(ab140601, abcam). The OVA12 antibody was prepared by our lab. To prepare the OVA12 antibody, we made a polyclonal antibody against the recombinant OVA12 full-length protein. The DNA sequence corresponding to the full-length protein was subcloned into the pET-32a (+) vector. Anti-OVA12 antisera were raised in rabbits against the purified HisOVA12 protein, and further affinity-purified on a protein A column.

\section{Real-time PCR analysis}

Total RNA was extracted from cultured cells using TRIzol reagent (Invitrogen) according to the manufacturer's instructions. First strand cDNA was synthesized using a Revert Aid First Strand cDNA synthesis kit (Fermentas). Real-time PCR was performed in a total volume of $20 \mu \mathrm{l}$ of reaction buffer. The primers 
used for amplification of the cDNA were synthesized by Sangon Biotech (Shanghai, China) (Table 2). Real-time PCR was performed using a SYBR Premix Ex Taq kit in an Applied Biosystems 7500 Fast Real-Time PCR system. The real-time PCR results were analyzed using the delta CT method.

\section{Luciferase reporter assay}

Siha-mock-shRNA/OVA12-shRNA, MCF-7-mockshRNA/OVA12-shRNA, and Caski-mock/OVA12 cells were plated in 6-well plates and co-transfected with 4.0 $\mu \mathrm{g}$ of the p53 reporter plasmid pp53-TA luc and $0.4 \mu \mathrm{g}$ of pRL-TK Renilla luciferase construct (Promega) as an internal control using Lipofectamine 2000. After a 48-h incubation, cell lysates were harvested in $500 \mu \mathrm{l}$ of Passive Lysis Buffer, and the p53 activity was determined with a luciferase assay system (Promega) according to the manufacturer's instructions. All experiments were performed in triplicate.

\section{Immunoprecipitation and ubiquitination assays}

Cells were treated with $10 \mu \mathrm{M}$ MG132 for 5 $\mathrm{h}$ before collection and then lysed in a lysis buffer (20 $\mathrm{mM}$ Tris $\mathrm{pH}$ 7.5, $150 \mathrm{mM} \mathrm{NaCl}, 1 \mathrm{mM}$ EDTA, $1 \mathrm{mM}$ EGTA, 1\% Triton X-100) supplemented with protease and phosphatase inhibitors (Thermo). Cell lysates were incubated with $2 \mu \mathrm{g}$ of p53 primary antibody overnight at $4{ }^{\circ} \mathrm{C}$. After incubation, $20 \mu \mathrm{l}$ of protein $\mathrm{A} / \mathrm{G}$ agarose beads (Thermo) were added to the cell lysates, and the mixture was incubated at $4{ }^{\circ} \mathrm{C}$ on a rotating platform for $3 \mathrm{~h}$. Then, the immunoprecipitates were collected by centrifugation at $1000 \times \mathrm{g}$ for $5 \mathrm{~min}$, and the supernatant was discarded. The beads were then washed 3 times with lysis buffer and dissolved in $100 \mu$ l of $2 \times$ loading buffer for Western blot analysis. The antibodies used in the coimmunoprecipitation experiments were anti-p53 and antiubiquitinantibodies.

\section{Protein half-life detection}

Siha-mock-shRNA/OVA12-shRNA and Caski-mock/ OVA12 cells were plated in 6-well plates and treated with $25 \mu \mathrm{g} / \mathrm{ml}$ cycloheximide ( $\mathrm{CHX}$ ) at the indicated time points. Cells were lysed in M-PER ${ }^{\mathrm{TM}}$ Mammalian Protein Extraction Reagent containing protease and phosphatase inhibitors after CHX treatment. Cell lysates were then collected for Western blotting to determine the $\mathrm{p} 53$ protein levels.

\section{Cell cycle assay}

Cells were trypsinized, rinsed twice with icecold PBS and fixed in 70\% ice-cold ethanol. Then, the cells were collected by centrifugation and resuspended in $0.2 \mathrm{mg} / \mathrm{ml}$ propidium iodide containing $0.1 \%$ Triton $\mathrm{X}-100$ and $1 \mathrm{mg} / \mathrm{ml}$ RNase A. The cell suspension was incubated in the dark for $30 \mathrm{~min}$ at $37^{\circ} \mathrm{C}$ and subsequently analyzed for DNA content using fluorescence-activated cell sorting (FACS) on a FACSCalibur flow cytometer (Becton Dickinson).

\section{Cell apoptosis assay}

Cells were plated in 6-well plates $24 \mathrm{~h}$ before induction of apoptosis. After treatment with cisplatin for $48 \mathrm{~h}$, the cells were harvested and double stained with annexin V-FITC and propidium iodide using an Annexin V-FITC Apoptosis Detection Kit (Becton Dickinson). Then, they were subjected to flow cytometric analysis and analyzed with the CELLQUEST software system. Cells in the right quadrant represented apoptotic cells.

\section{Statistical analysis}

All the obtained data are expressed as the means \pm standard deviation (SD). Statistical differences were evaluated with a two-tailed Student's t-test. $P$ values $<0.05$ were considered statistically significant. All statistical tests were performed using GraphPad Prism software version 5.01.

\section{ACKNOWLEDGMENTS}

We thank Dr. Xiaoren Zhang for his helpful discussion and support and Li Wang for technical assistance with the statistical analysis.

\section{CONFLICTS OF INTEREST}

The authors declare no conflicts of interest.

\section{GRANT SUPPORT}

This study was supported by grants from the National Natural Science Foundation of China (Nos. 30972786 and 81272328).

\section{REFERENCES}

1. Gjerstorff MF, Andersen MH, Ditzel HJ. Oncogenic cancer/ testis antigens: prime candidates for immunotherapy. Oncotarget. 2015; 6:15772-87. doi: 10.18632/ oncotarget.4694.

2. Caballero OL, Chen YT. Cancer/testis (CT) antigens: potential targets for immunotherapy. Cancer Sci. 2009; 100:2014-21.

3. Jin S, Wang Y, Zhang Y, Zhang HZ, Wang SJ, Tang JQ, Chen HJ, Ge HL. Humoral immune responses against tumor-associated antigen OVA66 originally defined by serological analysis of recombinant cDNA expression libraries and its potentiality in cellular immunity. Cancer Sci. 2008; 99:1670-78. 
4. Zhang R, Jin S, Rao W, Song F, Yin Q, Wang Y, Wang L, Xi Y, Zhang X, Wang M, Ge H. OVA12, a novel tumor antigen, promotes cancer cell growth and inhibits 5-fluorouracilinduced apoptosis. Cancer Lett. 2015; 357:141-51.

5. Levine AJ. p53, the cellular gatekeeper for growth and division. Cell. 1997; 88:323-31.

6. Ryan KM, Phillips AC, Vousden KH. Regulation and function of the p53 tumor suppressor protein. Curr Opin Cell Biol. 2001; 13:332-37.

7. Vogelstein B, Lane D, Levine AJ. Surfing the p53 network. Nature. 2000; 408:307-10.

8. Hupp TR, Lane DP, Ball KL. Strategies for manipulating the $\mathrm{p} 53$ pathway in the treatment of human cancer. Biochem J. 2000; 352:1-17.

9. Freed-Pastor WA, Prives C. Mutant p53: one name, many proteins. Genes Dev. 2012; 26:1268-86.

10. Muller PA, Vousden KH. Mutant p53 in cancer: new functions and therapeutic opportunities. Cancer Cell. 2014; 25:304-17.

11. Bieging KT, Mello SS, Attardi LD. Unravelling mechanisms of p53-mediated tumour suppression. Nat Rev Cancer. 2014; 14:359-70.

12. Duffy MJ, Synnott NC, McGowan PM, Crown J, O'Connor D, Gallagher WM. p53 as a target for the treatment of cancer. Cancer Treat Rev. 2014; 40:1153-60.

13. Kandoth C, McLellan MD, Vandin F, Ye K, Niu B, Lu C, Xie M, Zhang Q, McMichael JF, Wyczalkowski MA, Leiserson MD, Miller CA, Welch JS, et al. Mutational landscape and significance across 12 major cancer types. Nature. 2013; 502:333-39.

14. Kubbutat MH, Jones SN, Vousden KH. Regulation of p53 stability by Mdm2. Nature. 1997; 387:299-303.

15. Vassilev LT. MDM2 inhibitors for cancer therapy. Trends Mol Med. 2007; 13:23-31.

16. Honda R, Tanaka H, Yasuda H. Oncoprotein MDM2 is a ubiquitin ligase E3 for tumor suppressor p53. FEBS Lett. 1997; 420:25-27.

17. Wade M, Li YC, Wahl GM. MDM2, MDMX and p53 in oncogenesis and cancer therapy. Nat Rev Cancer. 2013; 13:83-96.

18. McCann AH, Kirley A, Carney DN, Corbally N, Magee HM, Keating G, Dervan PA. Amplification of the MDM2 gene in human breast cancer and its association with MDM2 and p53 protein status. Br J Cancer. 1995; 71:981-85.

19. Ho GH, Calvano JE, Bisogna M, Abouezzi Z, Borgen PI, Cordón-Cardó C, van Zee KJ. Genetic alterations of the p14ARF -hdm2-p53 regulatory pathway in breast carcinoma. Breast Cancer Res Treat. 2001; 65:225-32.

20. Pickart CM. Targeting of substrates to the $26 \mathrm{~S}$ proteasome. FASEB J. 1997; 11:1055-66.
21. Snoek BC, de Wilt LH, Jansen G, Peters GJ. Role of E3 ubiquitin ligases in lung cancer. World J Clin Oncol. 2013; 4:58-69.

22. Quelle DE, Zindy F, Ashmun RA, Sherr CJ. Alternative reading frames of the INK4a tumor suppressor gene encode two unrelated proteins capable of inducing cell cycle arrest. Cell. 1995; 83:993-1000.

23. Kamijo T, Zindy F, Roussel MF, Quelle DE, Downing JR, Ashmun RA, Grosveld G, Sherr CJ. Tumor suppression at the mouse INK4a locus mediated by the alternative reading frame product p19ARF. Cell. 1997; 91:649-59.

24. Stott FJ, Bates S, James MC, McConnell BB, Starborg M, Brookes S, Palmero I, Ryan K, Hara E, Vousden KH, Peters $\mathrm{G}$. The alternative product from the human CDKN2A locus, p14(ARF), participates in a regulatory feedback loop with p53 and MDM2. EMBO J. 1998; 17:5001-14.

25. Zhang Y, Xiong Y, Yarbrough WG. ARF promotes MDM2 degradation and stabilizes p53: ARF-INK4a locus deletion impairs both the $\mathrm{Rb}$ and $\mathrm{p} 53$ tumor suppression pathways. Cell. 1998; 92:725-34.

26. Pomerantz J, Schreiber-Agus N, Liégeois NJ, Silverman A, Alland L, Chin L, Potes J, Chen K, Orlow I, Lee HW, Cordon-Cardo C, DePinho RA. The Ink4a tumor suppressor gene product, $\mathrm{p} 19 \mathrm{Arf}$, interacts with MDM2 and neutralizes MDM2's inhibition of p53. Cell. 1998; 92:713-23.

27. Tao W, Levine AJ. P19(ARF) stabilizes p53 by blocking nucleo-cytoplasmic shuttling of Mdm2. Proc Natl Acad Sci USA. 1999; 96:6937-41.

28. Yang ZX, Wang D, Wang G, Zhang QH, Liu JM, Peng P, Liu XH. Clinical study of recombinant adenovirus-p53 combined with fractionated stereotactic radiotherapy for hepatocellular carcinoma. J Cancer Res Clin Oncol. 2010; 136:625-30.

29. Barone G, Tweddle DA, Shohet JM, Chesler L, Moreno L, Pearson AD, Van Maerken T. MDM2-p53 interaction in paediatric solid tumours: preclinical rationale, biomarkers and resistance. Curr Drug Targets. 2014; 15:114-23.

30. Nag S, Zhang X, Srivenugopal KS, Wang MH, Wang W, Zhang R. Targeting MDM2-p53 interaction for cancer therapy: are we there yet? Curr Med Chem. 2014; 21:553-74.

31. Vassilev LT, Vu BT, Graves B, Carvajal D, Podlaski F, Filipovic Z, Kong N, Kammlott U, Lukacs C, Klein C, Fotouhi N, Liu EA. In vivo activation of the p53 pathway by small-molecule antagonists of MDM2. Science. 2004; 303:844-48.

32. Saha MN, Qiu L, Chang H. Targeting p53 by small molecules in hematological malignancies. J Hematol Oncol. $2013 ; 6: 23$. 